Alberta Thy 09-05

PACS numbers: 04.50.+h, 98.80.Cq

August 2005

\title{
Integrability of Some Charged Rotating Supergravity Black Hole Solutions in Four and Five Dimensions
}

\author{
Muraari Vasudevan \\ Theoretical Physics Institute, University of Alberta, \\ Edmonton, Alberta T6G 2J1, Canada \\ E-mail: mvasudev@phys.ualberta.ca
}

\begin{abstract}
$\underline{\text { ABSTRACT }}$
We study the integrability of geodesic flow in the background of some recently discovered charged rotating solutions of supergravity in four and five dimensions. Specifically, we work with the gauged multicharge Taub-NUT-Kerr-(Anti) de Sitter metric in four dimensions, and the $U(1)^{3}$ gauged charged-Kerr-(Anti) de Sitter black hole solution of $\mathrm{N}=2$ supergravity in five dimensions. We explicitly construct the Killing tensors that permit separation of the Hamilton-Jacobi equation in these spacetimes. These results prove integrability for a large class of previously known supergravity solutions, including several BPS solitonic states. We also derive first-order equations of motion for particles in these backgrounds and examine some of their properties. Finally, we also examine the Klein-Gordon equation for a scalar field in these spacetimes and demonstrate separability.
\end{abstract}




\section{Introduction}

Solutions of the vacuum Einstein equations describing black hole solutions in both four and higher dimensions are currently of great interest. This is mainly due to a number of recent developments in high energy physics. Models of spacetimes with large extra dimensions have been proposed to deal with several questions arising in modern particle phenomenology (eat about.g. the hierarchy problem) [1-3]. These models allow for the existence of higher dimensional black holes which can be described classically. Also of interest in these models is the possibility of mini black hole production in high energy particle colliders which, if they occur, provide a window into non-perturbative gravitational physics $[4,5]$.

Superstring and M-Theory, which call for additional spacetime dimensions, naturally incorporate black hole solutions in higher dimensions (10 or 11). P-branes present in these theories can also support black holes, thereby making black hole solutions in an intermediate number of dimensions physically interesting as well. Black hole solutions in superstring theory are particularly relevant since they can be described as solitonic objects. They provide important keys to understanding strongly coupled non-perturbative phenomena which cannot be ignored at the Planck/string scale $[6,7]$.

Astrophysically relevant black hole spacetimes are, to a very good approximation, described by the Kerr metric [8]. One generalization of the Kerr metric to higher dimensions is given by the Myers-Perry construction [9]. With interest now in a nonzero cosmological constant, it is worth studying spacetimes describing rotating black holes with a cosmological constant. Another motivation for including a cosmological constant is driven by the AdS/CFT correspondence. The study of black holes in an Anti-de Sitter background could give rise to interesting descriptions in terms of the conformal field theory on the boundary leading to better understanding of the correspondence [10,11]. The general Kerr-de Sitter metrics describing rotating black holes in the presence of a cosmological constant have been constructed explicitly in $[12,13]$.

There is a strong need to understand explicitly the structure of geodesics in the background of black holes in Anti-de Sitter space in the context of string theory and the AdS/CFT correspondence. This is due to the recent work in exploring black hole singularity structure using geodesics and correlators in the dual CFT on the boundary [14-19]. The metrics mentioned above, have so far proven to yield little or no information through an analysis of this sort. Black holes with charge are particularly interesting for this type of analysis since the charges are reinterpreted as the R-charges of the dual theory. The spacetimes explored in this paper are exact solutions of supergravity in backgrounds with a 
cosmological constant and charges, and thus could be more suitable for this sort of geodesic analysis.

In this paper we work with the four-dimensional multicharge Kerr-Taub-NUT-(Anti) de Sitter solution of supergravity recently discovered by Chong, Cvetic, Lu, and Pope [20], as well as the $U(1)^{3}$ gauged Kerr-(Anti) de Sitter black hole solution of $\mathrm{N}=2$ supergravity in five dimensions discovered by Cvetic, Lu, and Pope [21].

We study the separability of the Hamilton-Jacobi equation in these spacetimes, which can be used to describe the motion of classical massive and massless particles (including photons). We use this explicit separation to obtain first-order equations of motion for both massive and massless particles in these backgrounds. The equations are obtained in a form that could be used for numerical study, and also in the study of black hole singularity structure using geodesic probes and the AdS/CFT correspondence.

We also study the Klein-Gordon equation describing the propagation of a massive scalar field in these spacetimes. Separation again turns out to be possible with the usual multiplicative ansatz.

This paper greatly generalizes the results of $[22,23]$ for the Myers-Perry metric in five dimensions, [24] which separates the equations in the case of equal rotation parameters in the odd dimensional Kerr-(A) dS spacetimes, [25] which separates the equations for the general five dimensional Kerr-(A) dS spacetime with unequal rotation parameters, [26] which separates the equations in the case of two independent sets of rotation parameters in the Myers-Perry metrics in all dimensions, [27] which separates the equations in the case of two independent sets of rotation parameters in the Kerr-(A) dS metrics in all dimensions, and [28] which separates the equations in the case of a single non-zero rotation parameter for uncharged Kerr-Taub-NUT metrics in arbitrary dimensions. Some further work for other special cases were also done in [29] and [30].

Separation turns out to be possible for both equations in these metrics due to the existence of second-order Killing tensors, one of them non-trivial and irreducible. This is a generalization of the Killing tensor in the Kerr black hole spacetime in four dimensions constructed in [31] which was subsequently described by Chandrasekhar as the "miraculous property of the Kerr metric". A similar construction for the Myers-Perry metrics in higher dimensions has also been done $[22,26]$, and for the Kerr-Taub-NUT metrics in arbitrary dimensions without charge and only one nonzero rotation parameter in [28]. The Killing tensors, in each case, provides an additional integral of motion necessary for complete integrability. 


\section{Overview of the Metrics}

\subsection{Four Dimensional Kerr-Taub-NUT Multicharge Gauged Solution of Supergravity}

This metric was recently obtained by Chong, Cvetic, Lu, and Pope in [20]. The solution was obtained by starting out with the four dimensional Kerr-Taub-NUT metric, dimensionally reducing to three dimensions along the time direction, and then lifting back up after "dualizing". The metric is given by

$$
d s^{2}=-\frac{\Delta_{r}}{a^{2} W}\left[a d t+u_{1} u_{2} d \phi\right]^{2}+\frac{\Delta_{u}}{a^{2} W}\left[a d t-r_{1} r_{2} d \phi\right]^{2}+W\left[\frac{d r^{2}}{\Delta_{r}}+\frac{d u^{2}}{\Delta_{u}}\right],
$$

where

$$
\begin{aligned}
W & =r_{1} r_{2}+u_{1} u_{2}, \quad r_{i}=r+2 m s_{i}^{2}, \quad u_{i}=u+2 l s_{i}^{2}, \quad i=1,2, \\
\Delta_{r} & =r^{2}+a^{2}-2 m r+g^{2} r_{1} r_{2}\left(r_{1} r_{2}+a^{2}\right), \\
\Delta_{u} & =-u^{2}+a^{2}+2 l u+g^{2} u_{1} u_{2}\left(u_{1} u_{2}-a^{2}\right)
\end{aligned}
$$

and we use the notation

$$
s_{i}=\sinh \delta_{i}, \quad c_{i}=\cosh \delta_{i}, \quad i=1,2 .
$$

Here $\delta_{1}$ is the magnetic charge, $\delta_{2}$ is the electric charge, $l$ is the NUT parameter, $a$ is the rotation parameter, and $g$ is the gauge parameter. The cosmological constant $\Lambda$ is given by $\Lambda=-g^{2}$. The ungauged solution is obtained by setting $g$ to zero.

If the two charge parameters are set equal, $\delta_{1}=\delta_{2}$, then the solution reduces to the charged AdS-Kerr-Taub-NUT solution of Einstein-Maxwell theory with a cosmological constant. To reduce to the usual coordinate system, we use the change of coordinate $u=a \cos \theta$. With $l$ set to zero, we recover the metric found in [20] for a multicharge Kerr-(Anti) de Sitter black hole in gauged supergravity in four dimensions.

For future reference, we note the following expressions. The determinant of the metric is given by

$$
g=-\frac{W^{2}}{a} .
$$

The components of the inverse metric are

$$
\begin{array}{rlrl}
g^{t t} & =\frac{1}{\Delta_{r} \Delta_{u} W}\left[\Delta_{r} u_{1}^{2} u_{2}^{2}-\Delta_{u} r_{1}^{2} r_{2}^{2}\right], & g^{\phi \phi} & =\frac{a^{2}}{\Delta_{r} \Delta_{u} W}\left[\Delta_{r}-\Delta_{u}\right], \\
g^{t \phi} & =\frac{a}{\Delta_{r} \Delta_{u} W}\left[\Delta_{r} u_{1} u_{2}+\Delta_{u} r_{1} r_{2}\right], & g^{r r}=\frac{\Delta_{r}}{W}, \quad g^{u u}=\frac{\Delta_{u}}{W} .
\end{array}
$$

We also note that the functions $\Delta_{r}$ and $\Delta_{u}$ are functions of $r$ and $u$ only, respectively. 


\section{$2.2 U(1)^{3}$ Gauged Kerr-(Anti) de Sitter Black Hole Solution of $\mathcal{N}=2$ Supergravity in Five Dimensions}

This metric was recently obtained by Chong, Lu, and Pope in [21]. The metric is given by

$$
\begin{aligned}
d s^{2}= & -\frac{Y-f_{3}}{R^{2}} d t^{2}+\frac{r^{2} R}{Y} d r^{2}+R d \Omega_{3}^{2}+\frac{f_{1}-R^{3}}{R^{2}}\left(\sin ^{2} \theta d \phi+\cos ^{2} \theta d \psi\right)^{2} \\
& -\frac{2 f_{2}}{R^{2}} d t\left(\sin ^{2} \theta d \phi+\cos ^{2} \theta d \psi\right)
\end{aligned}
$$

where

$$
\begin{aligned}
R & =r^{2}\left(\prod_{i=1}^{3} H_{i}\right)^{1 / 3}, \quad H_{i}=1+\frac{M s_{i}^{2}}{r^{2}} \\
d \Omega_{3}^{2} & =d \theta^{2}+\sin \theta^{2} d \phi^{2}+\cos ^{2} \theta d \psi^{2}
\end{aligned}
$$

and as before

$$
s_{i}=\sinh \delta_{i}, \quad c_{i}=\cosh \delta_{i}, \quad i=1,2,3 .
$$

The functions $f_{i}$, and $Y$ are defined by

$$
\begin{aligned}
f_{1}= & R^{3}+M a^{2} r^{2}+M^{2} a^{2}\left[2\left(\prod_{i} c_{i}-\prod_{i} s_{i}\right) \prod_{j} s_{j}-\sum_{i<j} s_{i}^{2} s_{j}^{2}\right], \\
f_{2}= & \gamma a \Lambda R^{3}+M a\left(\prod_{i} c_{i}-\prod_{i} s_{i}\right) r^{2}+M^{2} a \prod_{i} s_{i}, \\
f_{3}= & \gamma^{2} a^{2} \Lambda^{2} R^{3}+M a^{2} \Lambda\left[2 \gamma\left(\prod_{i} c_{i}-\prod_{i} s_{i}\right)-\Sigma\right] r^{2} \\
& +M a^{2}-\Lambda \Sigma M^{2} a^{2}\left[2\left(\left(\prod_{i} c_{i}-\prod_{i} s_{i}\right) \prod_{j} s_{j}-\sum_{i<j} s_{i}^{2} s_{j}^{2}\right]+2 \lambda \gamma M^{2} a^{2} \prod_{i} s_{i},\right. \\
Y= & f_{3}-\Lambda \Sigma R^{3}+r^{4}-M r^{2},
\end{aligned}
$$

and

$$
\Sigma=1+\gamma^{2} a^{2} \Lambda
$$

It is important to note that these are functions of the coordinate $r$ only.

The parameter $M$ is related to the mass of the black hole, the $\delta_{i}$ are the charges associated with each of the three $U(1)$ gauge groups, the gauge parameter $g$ is related to the cosmological constant $\Lambda$ via $\Lambda=-g^{2}, a$ is the rotation parameter of the black hole (equal rotation parameters in the two independent planes was assumed in the derivation of the 
metric), and the constant $\gamma$ is simply a redundant parameter which is useful to test several limits, but could be eliminated if necessary. This metric encompasses, as special limits, several previously known solutions such as the Klemm-Sabra BPS solution etc. More details about these limits can be found in [20].

In order to avoid long complicated expressions, we introduce the following functions to write the metric more compactly

$$
A(r)=\frac{Y-f_{3}}{R^{2}}, \quad W(r)=\frac{Y}{r^{2} R}, \quad B(r)=\frac{f_{1}-R^{3}}{R^{2}}, \quad C(r)=-\frac{f_{2}}{R^{2}} .
$$

Note that all of these are functions of the coordinate $r$ only. The metric is then written compactly in the form

$$
\begin{aligned}
d s^{2}= & -A(r) d t^{2}+\frac{d r^{2}}{W(r)}+R d \Omega_{3}^{2}+B(r)\left(\sin ^{2} \theta d \phi+\cos ^{2} \theta d \psi\right)^{2} \\
& +2 C(r) d t\left(\sin ^{2} \theta d \phi+\cos ^{2} \theta d \psi\right) .
\end{aligned}
$$

The components of the inverse metric are

$$
\begin{aligned}
g^{r r} & =W(r), \\
g^{\theta \theta} & =\frac{1}{R}, \\
g^{t t} & =-\frac{B(r)+R}{r^{2} W(r)}, \\
g^{t \phi} & =g^{t \psi}=\frac{C(r)}{r^{2} W(r)}, \\
g^{\phi \phi} & =\frac{A(r) B(r) \cos ^{2} \theta+A(r) R+C^{2}(r) \cos ^{2} \theta}{R r^{2} W(r) \sin ^{2} \theta}, \\
g^{\psi \psi} & =\frac{A(r) B(r) \sin ^{2} \theta+A(r) R+C^{2}(r) \sin ^{2} \theta}{R r^{2} W(r) \cos ^{2} \theta}, \\
g^{\phi \psi} & =-\frac{A(r) B(r)+C^{2}(r)}{R r^{2} W(r)} .
\end{aligned}
$$

We note for future reference the following identity which can easily be verified using Maple [32]

$$
A(r) B(r)+A(r) R+C^{2}(r)=r^{2} W(r) .
$$

Finally, the determinant of the metric can be calculated to be

$$
g=-r^{2} R^{2} \sin ^{2} \theta \cos ^{2} \theta
$$

where we need to make use of the identity given above repeatedly. 


\section{Integrals of Motion and the Hamilton-Jacobi Equation}

The equations of motion of a test particle of mass $m$ in a gravitational background described by a metric $g_{\mu \nu}$ are

$$
\frac{D^{2} x^{\mu}}{D \tau^{2}}=0
$$

where $\frac{D}{D \tau}$ is the covariant derivative with respect to proper time $\tau$. These equations can be derived from a Lagrangian

$$
L=\frac{1}{2} g_{\mu \nu} \dot{x^{\mu}} \dot{x^{\nu}}
$$

where a dot denotes a partial derivative with respect to an affine parameter $\lambda$. This can be chosen such that $\tau=m \lambda$.

The symmetries of the metric, if any, can provide us with some integrals of motion. For instance, if the metric is stationary, i.e. does not depend on the time $t$, then the energy is conserved. However, in most situations, sufficient number of integrals of motion do not exist. Also, using the Lagrangian formulation, sometimes certain integrals of motion are impossible to obtain even if they exist. Usually these are "second order" in the momenta such as the case of the Carter constant for the Kerr metric. Such additional integral of motion, which permit us, in these cases to completely integrate the equations of motion, can be provided by the Hamilton-Jacobi equation (though a proper choice of coordinate system is necessary).

The Hamilton-Jacobi equation in a curved background is given by

$$
-\frac{\partial S}{\partial \lambda}=H=\frac{1}{2} g^{\mu \nu} \frac{\partial S}{\partial x^{\mu}} \frac{\partial S}{\partial x^{\nu}}
$$

where $S$ is the action associated with the particle and $\lambda$ is some affine parameter along the worldline of the particle. Note that this treatment also accommodates the case of massless particles, where the trajectory cannot be parametrized by proper time.

\section{Particle Motion in the Four Dimensional Kerr-Taub-NUT Multicharge Gauged Solution of Supergravity}

\subsection{Separation of Variables}

We can attempt a separation of coordinates as follows. Let

$$
S=\frac{1}{2} m^{2} \lambda-E t+L_{\phi} \phi+S_{\theta}(\theta)+S_{r}(r) .
$$


$t$ and $\phi$ are cyclic coordinates, so their conjugate momenta are conserved. The conserved quantity associated with time translation is the energy $E$, and that with rotation in $\phi$ is the corresponding angular momentum $L_{\phi}$. Then using the components of the inverse metric (2.5), the Hamilton-Jacobi equation (3.3) is written to be

$$
\begin{aligned}
-m^{2}= & \frac{1}{\Delta_{r} \Delta_{u} W}\left[\Delta_{r} u_{1}^{2} u_{2}^{2}-\Delta_{u} r_{1}^{2} r_{2}^{2}\right](-E)^{2}+\frac{a^{2}}{\Delta_{r} \Delta_{u} W}\left[\Delta_{r}-\Delta_{u}\right] L_{\phi}^{2}+\frac{\Delta_{r}}{W}\left[\frac{d S_{r}(r)}{d r}\right]^{2} \\
& +\frac{\Delta_{u}}{W}\left[\frac{d S_{u}(u)}{d u}\right]^{2}+\frac{2 a}{\Delta_{r} \Delta_{u} W}\left[\Delta_{r} u_{1} u_{2}+\Delta_{u} r_{1} r_{2}\right](-E) L_{\phi}
\end{aligned}
$$

Now multiplying both sides by $W$, we can separate out the equation in the form

$$
\begin{aligned}
K & =\Delta_{r}\left[\frac{d S_{r}(r)}{d r}\right]^{2}-\frac{1}{\Delta_{r}}\left[r_{1} r_{2} E+a L_{\phi}\right]^{2}+m^{2} r_{1} r_{2}, \\
K & =-\Delta_{u}\left[\frac{d S_{u}(u)}{d u}\right]^{2}-\frac{1}{\Delta_{u}}\left[u_{1} u_{2} E-a L_{\phi}\right]^{2}-m^{2} u_{1} u_{2},
\end{aligned}
$$

where $K$ is a constant of separation.

\subsection{The Equations of Motion}

To derive the equations of motion, we will write the separated action $S$ from the HamiltonJacobi equation in the following form:

$$
S=\frac{1}{2} m^{2} \lambda-E t+L_{\phi} \phi+\int^{r} \sqrt{\mathcal{R}\left(r^{\prime}\right)} d r^{\prime}+\int^{u} \sqrt{U\left(u^{\prime}\right)} d u^{\prime},
$$

where

$$
\begin{aligned}
& \Delta_{r} \mathcal{R}(r)=K+\frac{1}{\Delta_{r}}\left[r_{1} r_{2} E+a L_{\phi}\right]^{2}-m^{2} r_{1} r_{2}, \\
& \Delta_{u} U(u)=-K-\frac{1}{\Delta_{u}}\left[u_{1} u_{2} E-a L_{\phi}\right]^{2}-m^{2} u_{1} u_{2} .
\end{aligned}
$$

To obtain the equations of motion, we differentiate $S$ with respect to the parameters $m^{2}, K, E, L_{\phi}$ and set these derivatives to equal other constants of motion. However, we can set all these new constants of motion to zero (following from freedom in choice of origin for the corresponding coordinates, or alternatively by changing the constants of integration). Following this procedure, we get the following equations of motion:

$$
\begin{aligned}
\frac{\partial S}{\partial m^{2}} & =0 \Rightarrow \lambda=\int r_{1} r_{2} \frac{d r}{\Delta_{r} \sqrt{\mathcal{R}}}+\int u_{1} u_{2} \frac{d u}{\Delta_{u} \sqrt{U}} \\
\frac{\partial S}{\partial K} & =0 \Rightarrow \int \frac{d u}{\Delta_{u} \sqrt{U}}=\int \frac{d r}{\Delta_{r} \sqrt{\mathcal{R}}} \\
\frac{\partial S}{\partial L_{\phi}} & =0 \Rightarrow \phi=\int\left(r_{1} r_{2} E+a L_{\phi}\right) \frac{d r}{\Delta_{r}^{2} \sqrt{\mathcal{R}}}+\int\left(u_{1} u_{2} E-a L_{\phi}\right) \frac{d u}{\Delta_{u}^{2} \sqrt{U}} \\
\frac{\partial S}{\partial E} & =0 \Rightarrow t=\int r_{1} r_{2}\left(r_{1} r_{2} E+a L_{\phi}\right) \frac{d r}{\Delta_{r}^{2} \sqrt{\mathcal{R}}}-\int u_{1} u_{2}\left(u_{1} u_{2} E-a L_{\phi}\right) \frac{d u}{\Delta_{u}^{2} \sqrt{U}}
\end{aligned}
$$


It is often more convenient to rewrite these in the form of first-order differential equations obtained from (4.6) by direct differentiation with respect to the affine parameter:

$$
\begin{aligned}
W \frac{d r}{d l} & =\Delta_{r} \sqrt{\mathcal{R}} \\
W \frac{d u}{d l} & =\Delta_{u} \sqrt{U} \\
W \frac{d \phi}{d l} & =\frac{r_{1} r_{2} E+a L_{\phi}}{\Delta_{r}}+\frac{u_{1} u_{2} E-a L_{\phi}}{\Delta_{\theta}} \\
W \frac{d t}{d l} & =\frac{r_{1} r_{2}\left(r_{1} r_{2} E+a L_{\phi}\right)}{\Delta_{r}}-\frac{u_{1} u_{2}\left(u_{1} u_{2} E-a L_{\phi}\right)}{\Delta_{\theta}} .
\end{aligned}
$$

\subsection{Analysis of the Radial Equation}

The worldline of particles in the background considered above are completely specified by the values of the conserved quantities $E, L_{\phi}, K$, and by the initial values of the coordinates. We will consider particle motion in the black hole exterior. Allowed regions of particle motion necessarily need to have positive value for the quantity $R$, owing to equation (4.7). We determine some of the possibilities of the allowed motion.

We will consider the motion of a particle in the black hole exterior. Thus we can assume that $\Delta_{r}>0$ for large $r$. At large $r$, the dominant contribution to $\mathcal{R}$, in the case of $\Lambda=0$, is $E^{2}-m^{2}$. Since $\Lambda=-g^{2}$, zero cosmological constant corresponds to the charged rotating black hole in four dimensions in ungauged supergravity. Here, we can thus say that for $E^{2}<m^{2}$, we cannot have unbounded orbits, whereas for $E^{2}>m^{2}$, such orbits are possible. For the case of nonzero $\Lambda$ (i.e. also nonzero $g$ which implies we are now considering gauged supergravity), the dominant term at large $r$ in $R$ (or rather the slowest decaying term) is $-m^{2} r^{2}$. Thus in the case of the Anti-de Sitter background (since $\Lambda=-g^{2}$ is negative), only bound orbits are possible.

In order to study the radial motion of particles in these metrics, it is useful to cast the radial equation of motion into a different form. Decompose $\mathcal{R}$ as a quadratic in $E$ as follows:

$$
\mathcal{R}=\alpha E^{2}-2 \beta E+\gamma
$$

where

$$
\begin{aligned}
\alpha & =\frac{r_{1}^{2} r_{2}^{2}}{\Delta_{r}^{2}} \\
\beta & =-\frac{r_{1} r_{2} a L_{\phi}}{\Delta_{r}^{2}} \\
\gamma & =\frac{K-m^{2} r_{1} r_{2}}{\Delta_{r}}+\frac{a^{2} L_{\phi}^{2}}{\Delta_{r}^{2}}
\end{aligned}
$$


The turning points for trajectories in the radial motion (defined by the condition $\mathcal{R}=0$ ) are given by $E=V_{ \pm}$where

$$
V_{ \pm}=\frac{\beta \pm \sqrt{\beta^{2}-\alpha \gamma}}{\alpha}
$$

These functions, called the effective potentials [22], determine allowed regions of motion. In this form, the radial equation is much more suitable for detailed numerical analysis for specific values of parameters.

\section{Particle Motion in the $U(1)^{3}$ Gauged Kerr-(Anti) de Sitter Black Hole Solution of $\mathcal{N}=2$ Supergravity in Five Dimen- sions}

We will only sketch the analysis of the separation of variables here, since the procedure for deriving equations of motion etc. is virtually identical to those of the metric above.

We can attempt a separation of coordinates as follows. Let

$$
S=\frac{1}{2} m^{2} \lambda-E t+L_{\phi} \phi+L_{\psi} \psi+S_{\theta}(\theta)+S_{r}(r)
$$

$t, \phi$, and $\psi$ are cyclic coordinates, so their conjugate momenta are conserved. The conserved quantity associated with time translation is the energy $E$, and those with rotation in $\phi$ and $\psi$ are the corresponding angular momenta $L_{\phi}$ and $L_{\psi}$. Then using the components of the inverse metric (2.13), the Hamilton-Jacobi equation (3.3) is written to be

$$
\begin{aligned}
-m^{2} & =\frac{B(r)+R}{r^{2} W(r)}(-E)^{2}+2 \frac{C(r)}{r^{2} W(r)}(-E)\left(L_{\phi}\right)+2 \frac{C(r)}{r^{2} W(r)}(-E)\left(L_{\psi}\right) \\
& +\frac{A(r) B(r) \cos ^{2} \theta+A(r) R+C^{2}(r) \cos ^{2} \theta}{R r^{2} W(r) \sin ^{2} \theta} L_{\phi}^{2}+\frac{1}{R}\left[\frac{d S_{\theta}(\theta)}{d \theta}\right]^{2}+ \\
& +\frac{A(r) B(r) \sin ^{2} \theta A(r) R+C^{2}(r) \sin ^{2} \theta}{R r^{2} W(r) \cos ^{2} \theta} L_{\psi}^{2}+W(r)\left[\frac{d S_{r}(r)}{d r}\right]^{2}
\end{aligned}
$$

After some algebraic manipulation and using some trigonometric identites we can write this as

$$
\begin{aligned}
-m^{2} & =W(r)\left[\frac{d S_{r}(r)}{d r}\right]^{2}-2 \frac{C(r) E}{r^{2} W(r)}\left(L_{\phi}+L_{\psi}\right)-\frac{B(r)+R}{r^{2} W(r)} E^{2} \\
& -\frac{A(r) B(r)+C^{2}(r)}{R r^{2} W(r)}\left(L_{\phi}+L_{\psi}\right)^{2}+\frac{1}{R}\left(\csc ^{2} \theta L_{\phi}^{2}+\sec ^{2} \theta L_{\psi}^{2}\right)+\frac{1}{R}\left[\frac{d S_{\theta}(\theta)}{d \theta}\right]^{2}(5.3
\end{aligned}
$$

In this form, the Hamilton-Jacobi equation can now be easily separated to give

$$
-K=\left[\frac{d S_{\theta}(\theta)}{d \theta}\right]^{2}+\csc ^{2} \theta L_{\phi}^{2}+\sec ^{2} \theta L_{\psi}^{2},
$$




$$
\begin{aligned}
K & =R m^{2}+W(r) R\left[\frac{d S_{r}(r)}{d r}\right]^{2}-2 \frac{C(r) R E}{r^{2} W(r)}\left(L_{\phi}+L_{\psi}\right)-\frac{B(r) R+R^{2}}{r^{2} W(r)} E^{2} \\
& -\frac{A(r) B(r)+C^{2}(r)}{r^{2} W(r)}\left(L_{\phi}+L_{\psi}\right)^{2}
\end{aligned}
$$

where $K$ is a constant of separation.

To derive the equations of motion, the separated action $S$ from the Hamilton-Jacobi equation is more conveniently written, as before, in the following form:

$$
S=\frac{1}{2} m^{2} \lambda-E t+L_{\phi} \phi+L_{\psi} \psi+\int^{r} \sqrt{\mathcal{R}\left(r^{\prime}\right)} d r^{\prime}+\int^{\theta} \sqrt{\Theta\left(\theta^{\prime}\right)} d \theta^{\prime},
$$

where

$$
\begin{aligned}
R W(r) \mathcal{R}(r) & =K-R m^{2}+2 \frac{C(r) R E}{r^{2} W(r)}\left(L_{\phi}+L_{\psi}\right)+\frac{B(r) R+R^{2}}{r^{2} W(r)} E^{2} \\
& +\frac{A(r) B(r)+C^{2}(r)}{r^{2} W(r)}\left(L_{\phi}+L_{\psi}\right)^{2} \\
\Theta(\theta) & =-K-\csc ^{2} \theta L_{\phi}^{2}-\sec ^{2} \theta L_{\psi}^{2} .
\end{aligned}
$$

By following the same procedure as earlier, we can easily establish first order equations of motion, and a radial effective potential etc. Since the derivation is remarkably similar, we will not reproduce the results here in the interests of being concise.

\section{Dynamical Symmetry}

The general class of metrics discussed here are stationary and "axisymmetric"; i.e., $\partial / \partial t$ and $\partial / \partial \phi$ (as well as $\partial / \partial \psi$ in the five dimensional $U(1)^{3}$ case) are Killing vectors and have associated conserved quantities, $-E$ and $L_{\phi}$ (and $L_{\psi}$ ). In general if $\xi$ is a Killing vector, then $\xi^{\mu} p_{\mu}$ is a conserved quantity, where $p$ is the momentum of the particle. Note that this quantity is first order in the momenta.

As mentioned earlier, the additional constant of motion $K$ which allowed for complete integrability of the equations of motion is not related to a Killing vector from a cyclic coordinate. This constant is, rather, derived from a non-trivial irreducible second-order Killing tensor in both spacetimes, which permits the separation of the $r-\theta$ (or $r-u$ ) equations in both cases. These Killing tensors are generalizations of the Killing tensor obtained in four dimensions by Carter [31] and in five dimensions for the Myers-Perry metric in [22]. Killing tensors are not symmetries on configuration space, and cannot be derived from a Noether procedure, and are rather, symmetries on phase space. They obey 
a generalization of the Killing equation for Killing vectors (which do generate symmetries in configuration space by the Noether procedure) given by

$$
K_{(\mu \nu ; \rho)}=0
$$

where $K$ is any second order Killing tensor, and the parentheses indicate complete symmetrization of all indices.

The Killing tensors can be obtained from the expressions for the separation constant $K$ in each case. If the particle has momentum $p$, then the Killing tensor $\mathcal{K}_{\mu \nu}$ is related to the constant $K$ via

$$
K=\mathcal{K}^{\mu \nu} p_{\mu} p_{\nu}=\mathcal{K}^{\mu \nu} \frac{\partial S}{\partial x^{\mu}} \frac{\partial S}{\partial x^{\nu}}
$$

In both cases, we can use the expression in terms of the $r$ equation or the $u / \theta$ equation. We will choose to work with the latter in both cases.

For the four dimensional Kerr-Taub-NUT metric analyzed above, the expression for $K$ from (4.3) is

$$
K=-\Delta_{u}\left[\frac{d S_{u}(u)}{d u}\right]^{2}-\frac{1}{\Delta_{u}}\left[u_{1} u_{2} E-a L_{\phi}\right]^{2}-m^{2} u_{1} u_{2} .
$$

Thus, from (6.2) we can easily read

$$
\mathcal{K}=-\Delta_{u} \partial_{u} \otimes \partial_{u}-\frac{1}{\Delta_{u}}\left[u_{1} u_{2} \partial_{t} \otimes \partial_{t}+a^{2} \partial_{\phi} \otimes \partial_{\phi}+\operatorname{sym}\left(a u_{1} u_{2} \partial_{t} \otimes \partial_{\phi}\right)\right]
$$

Since this Killing tensor is not a simple linear combination of Killing vectors, it is nontrivial and irreducible.

For the five dimensional $U(1)^{3}$ charged metric analyzed above, the expressions for $K$ from (5.4) is

$$
-K=\left[\frac{d S_{\theta}(\theta)}{d \theta}\right]^{2}+\csc ^{2} \theta L_{\phi}^{2}+\sec ^{2} \theta L_{\psi}^{2}
$$

Thus, again from (6.2) we can read

$$
\mathcal{K}=-\partial_{\theta} \otimes \partial_{\theta}-\frac{1}{\sin ^{2} \theta} \partial_{\phi} \otimes \partial_{\phi}-\frac{1}{\cos ^{2} \theta} \partial_{\psi} \otimes \partial_{\psi} .
$$

This Killing tensor however turns out to be a reducible one. In this situation, since both rotation parameters, there is an additional Killing vector which represents the additional symmetry of being able to rotate each of the two rotation planes into each other. This Killing tensor can be obtained using linear combinations of outer products of this Killing vector. Further details and explicit constructions can be found in [26].

We can easily check using Maple [32], that the Killing tensors in both spacetimes do satisfy the Killing equation. It is the existence of these Killing tensors that allows for complete separation of the Hamilton-Jacobi equation. 


\section{The Scalar Field Equation}

Consider a scalar field $\Psi$ in a gravitational background with the action

$$
S[\Psi]=-\frac{1}{2} \int d^{D} x \sqrt{-g}\left((\nabla \Psi)^{2}+\alpha R \Psi^{2}+m^{2} \Psi^{2}\right),
$$

where we have included a curvature dependent coupling. However, in these Kerr-(Anti) de Sitter backgrounds with charges, $R$ is constant (proportional to the cosmological constant $\Lambda)$. As a result we can trade off the curvature coupling for a different mass term. So it is sufficient to study the massive Klein-Gordon equation in this background. We will simply set $\alpha=0$ in the following. Variation of the action leads to the Klein-Gordon equation

$$
\frac{1}{\sqrt{-g}} \partial_{\mu}\left(\sqrt{-g} g^{\mu \nu} \partial_{\nu} \Psi\right)=m^{2} \Psi
$$

\subsection{Massive Scalar Fields in the Four Dimensional Kerr-Taub-NUT Mul- ticharge Gauged Solution of Supergravity}

Using the explicit expressions for the components of the inverse metric (2.5) and the determinant (2.4), the Klein-Gordon equation for a massive scalar field in this spacetime can be written as

$$
\begin{aligned}
m^{2} \Psi & =\frac{1}{W}\left(\frac{1}{\Delta_{u} \Delta_{r}}\left[\Delta_{r} u_{1}^{2} u_{2}^{2}-\Delta_{u} r_{1}^{2} r_{2}^{2}\right] \partial_{t}^{2} \Psi+\frac{2 a}{\Delta_{r} \Delta_{u}}\left[\Delta_{r} u_{1} u_{2}+\Delta_{u} r_{1} r_{2}\right] \partial_{t \phi}^{2} \Psi\right. \\
& \left.+\frac{2 a^{2}}{\Delta_{r} \Delta_{u}}\left[\Delta_{r}-\Delta_{u}\right] \partial_{\phi}^{2} \Psi+\partial_{r}\left(\Delta_{r} \partial_{r} \Psi\right)+\partial_{u}\left(\Delta_{u} \partial_{u} \Psi\right)\right) .
\end{aligned}
$$

(Note that this expression agrees with equation (16) in [28] with the notation $p=a \cos \theta$, $q=r, X=\Delta_{u}, Y=\Delta_{r}$, and $k=0$ in four dimensions for the uncharged, i.e. $\delta_{i}=0$, Kerr-Taub-NUT metrics. This is a good check for consistency.) We assume the usual multiplicative ansatz for the separation of the Klein-Gordon equation

$$
\Psi=\Phi_{r}(r) \Phi_{u}(u) e^{-i E t} e^{i L_{\phi} \phi}
$$

Then we can easily separate out the $r$ and $u$ dependance as

$$
\begin{aligned}
K & =-\frac{1}{\Phi_{u}(u)} \frac{d}{d u}\left(\Delta_{u} \frac{d \Phi_{u}(u)}{d u}\right)+m^{2} u_{1} u_{2}+\frac{u_{1}^{2} u_{2}^{2}}{\Delta_{u}} E^{2}-\frac{2 a u_{1} u_{2}}{\Delta_{u}} E L_{\phi}+\frac{2 a^{2} L_{\phi}^{2}}{\Delta_{u}} \\
K & =\frac{1}{\Phi_{r}(r)} \frac{d}{d r}\left(\Delta_{r} \frac{d \Phi_{r}(r)}{d r}\right)-m^{2} r_{1} r_{2}-\frac{r_{1}^{2} r_{2}^{2}}{\Delta_{r}} E^{2}+\frac{2 a r_{1} r_{2}}{\Delta_{r}} E L_{\phi}+\frac{2 a^{2} L_{\phi}^{2}}{\Delta_{r}}
\end{aligned}
$$

where $K$ is again a separation constant. At this point we have completely separated out the Klein-Gordon equation for a massive scalar field in this spacetime. 


\subsection{Massive Scalar Fields in the $U(1)^{3}$ Gauged Kerr-(Anti) de Sitter Black Hole Solution of $\mathcal{N}=2$ Supergravity in Five Dimensions}

Using the explicit expressions for the components of the inverse metric (2.13) and the determinant (2.15), the Klein-Gordon equation for a massive scalar field in this spacetime can be written as

$$
\begin{aligned}
m^{2} \Psi & =-\frac{B(r)+R}{r^{2} W(r)} \partial_{t}^{2} \Psi+\frac{2 C(r)}{r^{2} W(r)}\left(\partial_{t \phi}^{2} \Psi+\partial_{t \psi} \Psi\right)+\frac{1}{r R} \partial_{r}\left(r R W(r) \partial_{r} \Psi\right) \\
& +\frac{A(r) B(r) \cos ^{2} \theta+A(r) R+C^{2}(r) \cos ^{2} \theta}{R \sin ^{2} \theta r^{2} W(r)} \partial_{\phi}^{2} \Psi \\
& +\frac{A(r) B(r) \sin ^{2} \theta+A(r) R+C^{2}(r) \sin ^{2} \theta}{R \cos ^{2} \theta r^{2} W(r)} \partial_{\psi}^{2} \Psi-\frac{2\left[A(r) B(r)+C^{2}(r)\right]}{R r^{2} W(r)} \partial_{\phi \psi}^{2} \Psi \\
& +\frac{1}{R \sin \theta \cos \theta} \partial_{\theta}\left(\sin \theta \cos \theta \partial_{\theta} \Psi\right) .
\end{aligned}
$$

Again we assume the usual multiplicative ansatz for separation

$$
\Psi=\Phi_{r}(r) \Phi_{\theta}(\theta) e^{-i E t} e^{i L_{\phi} \phi} e^{i L_{\psi} \psi}
$$

After extensive algebraic manipulation similar to that of the Hamilton-Jacobi equation, and the use of some trigonometric identities along the way, we find that the $r$ and $\theta$ equations decouple into the form

$$
\begin{aligned}
K & =-\frac{1}{\Phi_{\theta}(\theta) \sin \theta \cos \theta} \frac{d}{d \theta}\left(\sin \theta \cos \theta \frac{d \Phi_{\theta}(\theta)}{d \theta}\right)+\frac{L_{\phi}^{2}}{\sin ^{2} \theta}+\frac{L_{\psi}^{2}}{\cos ^{2} \theta} \\
K & =\frac{1}{\Phi_{r}(r) r} \frac{d}{d r}\left(r R W(r) \frac{d \Phi_{r}(r)}{d r}\right)+\frac{B(r) R+R^{2}}{r^{2} W(r)} E^{2}+\frac{2 C(r) R}{r^{2} W(r)}\left(L_{\phi}+L_{\psi}\right) \\
& +\frac{A(r) B(r)+C^{2}(r)}{r^{2} W(r)}\left(L_{\phi}+L_{\psi}\right)^{2}
\end{aligned}
$$

where $K$ is again a separation constant. At this point we have completely separated out the Klein-Gordon equation for a massive scalar field in this spacetime.

We note the role of the Killing tensors in the separation terms of the Klein-Gordon equations in both spacetimes. In fact, the complete integrability of geodesic flow of both metrics via the Hamilton-Jacobi equation can be viewed as the classical limit of the statement that the Klein-Gordon equation in both metrics also completely separates.

\section{Conclusions}

We studied the complete integrability properties of the Hamilton-Jacobi and the KleinGordon equations in the background of two recently discovered rotating black hole solutions 
of supergravity with charge(s): the four dimensional Kerr-Taub-NUT Multicharge gauged supergravity solution, and the $U(1)^{3}$ gauged Kerr-(Anti) de Sitter black hole solution of $\mathcal{N}=2$ supergravity in five dimensions. Complete separation of both the Hamilton-Jacobi and Klein-Gordon equations in these backgrounds in Boyer-Lindquist-like coordinates is demonstrated. This is due to the enlarged dynamical symmetry of the spacetime. We construct the Killing tensors (one of them irreducible) in both spacetimes which explicitly permits complete separation. We also derive first-order equations of motion for classical particles in these backgrounds, and analyze the properties of some special trajectories. It should be emphasized that these complete integrability properties are a fairly non-trivial consequence of the specific form of the metrics, and generalize several such remarkable properties for other previously known metrics.

Further work in this direction could include the study of higher-spin field equations in these backgrounds, which is of great interest, particularly in the context of string theory. Explicit numerical study of the equations of motion for specific values of the black hole parameters could lead to interesting results. The geodesic equations presented can also readily be used in the study of black hole singularity structure in an AdS background using the AdS/CFT correspondence.

\section{References}

[1] N. Arkani-Ahmed, S Dimopoulos and G. Dvali The Hierarchy Problem and new dimensions at a millimeter, Phys.Lett. B429 (1998) 263-272, hep-ph/9803315.

[2] I. Antoniadis, N. Arkani-Hamed, S. Dimopoulos and G. Dvali New dimensions at a millimeter to a Fermi and superstrings at a TeV, Phys.Lett. B436 (1998) 257-263, hep-ph/9804398

[3] L. Randall and R. Sundrum A large mass hierarchy from a small extra dimension, Phys.Rev.Lett. 83 (1999) 3370-3373, hep-ph/9905221.

[4] M. Cavaglia Black hole and brane production in TeV gravity: A review, Int.J.Mod.Phys. A18 (2003) 1843-1882, hep-ph/0210296.

[5] P. Kanti Black holes in theories with large extra dimensions: a Review, hep-ph/0402168.

[6] G. Dvali and A. Vilenkin Solitonic D-branes and brane annihilation, Phys.Rev. D67 (2003) 046002, hep-th/0209217. 
[7] M. Cvetic and A. A. Tseytlin Solitonic strings and BPS saturated dyonic black holes, Phys.Rev. D53 (1996) 5619-5633, hep-th/9512031.

[8] R.P. Kerr, Gravitational field of a spinning mass as an example of algebraically special metrics, Phys. Rev. Lett. 11, 237 (1963).

[9] R.C. Myers and M.J. Perry, Black holes in higher dimensional space-times, Ann. Phys. 172, 304 (1986).

[10] J. Maldacena The large $N$ limit of superconformal field theories and supergravity, Adv.Theor.Math.Phys. 2 (1998) 231-252; Int.J.Theor.Phys. 38 (1999) 1113-1133, hep-th/9711200.

[11] E. Witten Anti de Sitter space And holography, Adv.Theor.Math.Phys. 2 (1998) 253-29, hep-th/9802150.

[12] G.W. Gibbons, H. Lü, D.N. Page and C.N. Pope, The general Kerr-de Sitter metrics in all dimensions, J. Geom. Phys. 53 (2005) 49-73, hep-th/0404008.

[13] G.W. Gibbons, H. Lü, D.N. Page and C.N. Pope, Rotating black holes in higher dimensions with a cosmological constant, Phys. Rev. Lett. 93:171102 (2004) 49-73, hep-th/0409155.

[14] L. Fidkowski, V. Hubeny, M. Kleban and S. Shenker, The black hole singularity in AdS/CFT, JHEP 0402 (2004) 014, hep-th/0306170

[15] D. Brecher, J. He and M. Razali, On charged black holes in anti-de Sitter space, JHEP 0504 (2005) 004, hep-th/0410214.

[16] N. Cruz, M. Olivares, J. Villanueva, The geodesic structure of the Schwarzchild anti-de Sitter black hole, Class. Quant. Grav 22 (2005) 1167-1190, gr-qc/0408016.

[17] J. Kaplan, Extracting data from behind horizons with the AdS/CFT correspondence, hep-th/0402066.

[18] V. Hubeny, Black hole singularity in AdS/CFT, hep-th/0401138.

[19] V. Balasubramanian and T.S. Levi, Beyond the veil: inner horizon instability and holography, Phys. Rev. D70 (2004) 106005, hep-th/0405048. 
[20] Z.W. Chong, M. Cvetic, H. Lu and C.N. Pope, Charged rotating black holes in fourdimensional gauged and ungauged supergravities, Nucl.Phys. B717 (2005) 246-271, hep-th/0411045.

[21] M. Cvetic, H. Lu and C.N. Pope, Charged rotating black holes in five dimensional $U(1)^{3}$ gauged N=2 supergravity, Phys.Rev. D70 (2004) 081502, hep-th/0407058.

[22] V. Frolov and D. Stojkovic, Particle and light motion in a space-time of a fivedimensional rotating black hole, Phys.Rev. D68 (2003) 064011, gr-qc/0301016.

[23] V. Frolov and D. Stojkovic, Quantum radiation from a 5-dimensional rotating black hole, Phys.Rev. D67 (2003) 084004, gr-qc/0211055.

[24] M. Vasudevan, K. Stevens and D.N. Page, Separability of the Hamilton-Jacobi and Klein-Gordon equations in Kerr-de Sitter metrics, Class. Quantum Grav. 22 (2005) 14691482, gr-qc/0407030.

[25] H.K. Kunduri and J. Lucietti, Integrability and the Kerr-(A)dS black hole in five dimensions, Phys. Rev. D71 (2005) 104021, hep-th/0502124.

[26] M. Vasudevan, K. Stevens and D.N. Page, Particle motion and scalar field propogation in Myers-Perry black hole spacetimes in all dimensions, Class. Quantum Grav. 22 (2005) 339352, gr-qc/0405125

[27] M. Vasudevan and K. Stevens, Integrability of particle motion and scalar field propagation in Kerr-(Anti) de Sitter black hole spacetimes in all dimensions, gr-qc/0507096.

[28] Z.W. Chong, G.W. Gibbons, H. Lu and C.N. Pope, Separability and Killing tensors in Kerr-Taub-NUT-de Sitter metrics in higher dimensions, Phys.Lett. B609 (2005) 124-132, hep-th/0405061.

[29] M.M. Caldarelli, D. Klemm and W.A. Sabra Causality violation and naked time machines in AdS5, JHEP 0105 (2001) 014, hep-th/0103133.

[30] H.K. Kunduri and J. Lucietti, Notes on non-extremal, charged, rotating black holes in minimal D=5 gauged supergravity, Nucl. Phys. B724 (2005) 343-356, hep-th/0504158.

[31] B. Carter, Hamilton-Jacobi and Schrodinger separable solutions of Einstein's equations, Commun. Math. Phys. 10, 280 (1968).

[32] Maple 6 for Linux, Maplesoft Inc., Waterloo Ontario, http://www.maplesoft.com. 